\title{
General practitioner referral patterns for women with gynaecological symptoms: a randomised incomplete block study design
}

\section{Shanthi A \\ Ramanathan \\ BA, MHlthSci(Hons) Senior Research Fellow \\ Genevieve Baratiny BSc(Hons), PhD Research Fellow ${ }^{2}$ \\ Nigel P Stock MD, FRACGP, FAFPHM Head and Professor of General Practice ${ }^{3}$ \\ Andrew M Searles BEc, MMedStats, PhD, Hunter Valley Research Foundation-Hunter Medical Research Institute Health-Research Economist \\ Russell J Redford BSc, DipCompSc, Statistician/Programmer \\ 1 Hunter Valley Research Foundation Newcastle, NSW. \\ 2 Centre for Rural Health and Community Development, University of South Australia, Whyalla, SA. \\ 3 School of Population Health and Clinical Practice University of Adelaide Adelaide, SA. \\ shanthi@hvrf.com.au}

MJA 2011; 195: 602-606 doi:10.5694/mjal0.10867 n Australia, more than 4000 women are diagnosed each year with a gynaecological cancer; one in 33 Australian women will be diagnosed with a gynaecological cancer before the age of 75 years. ${ }^{1}$ Delays in the diagnosis of such cancers are a major cause of poor outcomes. For example, ovarian cancer has a poor prognosis, because many cases are diagnosed at an advanced stage., ${ }^{2,3}$ Differences in access to health care and quality diagnostic care are primary contributors to differences in diagnosis, survival rates, and mortality outcomes for women with cervical cancer. $^{4}$

In Australia, general practitioners provide the bulk of primary health care, and are often the first health care professionals visited by women experiencing gynaecological symptoms. GPs refer patients to specialists for diagnosis, investigation, treatment and reassurance, as a result of patient pressure, to obtain a second opinion, and for medicolegal reasons. ${ }^{5}$ Patients gain access to specialists like gynaecologists through referral from GPs, who refer to specialists at an overall rate of $7.7 \%$, with referrals to gynaecologists ranking 6 th most common and comprising $4.6 \%$ of all medical referrals. ${ }^{6}$

Our aim in this survey was to collect national baseline data to establish referral practices for women with symptoms indicative of gynaecological cancers. Such data would assist in evaluating the effectiveness of the strategies implemented by Cancer Australia and the National Centre for Gynaecological Cancers (NCGC) to reduce variability in the diagnosis and management of these gynaecological cancers.

The survey was designed to:

- identify and distinguish why, when and to whom GPs refer women with symptoms indicative of gynaecological cancer;

- identify patient and GP factors that predict referral; and

\section{Abstract}

Objective: To describe why, when and to whom general practitioners refer women with symptoms possibly attributable to cervical, endometrial or ovarian cancers, and to identify patient and GP factors that predict referral to either a gynaecologist or a gynaecological oncologist.

Design and setting: A national survey of GPs between 1 April and 31 August 2009 using a randomised incomplete block design based on case vignettes, and using a self-completed postal or online questionnaire.

Participants: A sample of GPs, stratified by location and randomly selected from a database of GPs maintained by the Australasian Medical Publishing Company.

Main outcome measures: Proportion of vignettes that were deemed to reflect a high probability of cancer being referred; and the patient and clinician factors that were the strongest predictors of referral.

Results: Of the 3082 GPs who were selected for participation, 1402 responded, giving a response rate of $45.5 \%$. Overall, for vignettes identified as describing women with a high probability of cancer, $75 \%$ were referred by metropolitan GPs and $73 \%$ by rural practitioners. Metropolitan GPs were significantly more likely to refer women in scenarios indicative of endometrial cancer than rural GPs. For all three cancers, GPs were significantly more likely to refer a patient to a gynaecologist (between 70.8\% and 95.4\%) than a gynaecological oncologist. Metropolitan GPs had significantly greater access to both private and public gynaecological oncologists than their rural counterparts. Referral rates were higher for ovarian and cervical cancer ( $83 \%$ and $80 \%$, respectively) and lower for endometrial cancer (68\%). For all three cancers, patient factors were stronger predictors of referral than the demographic factors of participating GPs.

Conclusion: There appears to be significant variation in referral practices among GPs and this variation is greater for endometrial cancer, for which there are currently no evidence-based clinical practice guidelines in Australia. There is a need for further research into understanding the basis of these differences, including a review of the existing guidelines for ovarian and cervical cancer and the development of guidelines for endometrial cancer.

- inform recommendations improve clinical practice.

\section{Methods}

\section{Design and setting}

The design of the study was developed as part of a separate pilot conducted by researchers from Monash University (a 2008 unpublished report on patterns of care in gynaecological cancers commissioned by the NCGC). Another study was used as the basis for developing the study methods. ${ }^{7}$ The Monash team undertook an extensive review of evidence-based clinical practice guidelines relevant to referral behaviour for ovarian, cervical and endometrial cancers, which informed the development of the set to of vignettes for this study. Their extensive search revealed a paucity of evidence to guide clinical practice, including referral. ${ }^{7-9}$ Six clinical practice guidelines were relevant (two to ovarian cancer, three to cervical cancer and one to all gynaecological cancers) and were reviewed. In most instances, the recommendations were based on low-level evidence or on expert opinion. The information derived from the clinical practice guidelines was used to inform the content of the study vignettes and was supplemented with expert opinion.

Thirty-one clinicians (12 GPs, 12 gynaecologists and seven gynaecological oncologists) participated in telephone interviews to elicit their views about clinical factors influencing referral of women with suspected 
gynaecological cancers, and to discuss issues associated with variations in their care. The resulting clinical variables were added to the clinical practice guideline recommendations and prioritised to form a pool of fixed (contextual) factors and clinical variables relevant to each cancer type.

\section{Vignettes}

We used a series of vignettes, each containing a hypothetical scenario about a patient with gynaecological symptoms. Vignettes have the advantage of reflecting real-life situations in a concise manner, while controlling for external, potentially confounding variables and contextual variations between patients. They are recognised as valid, simple and cost-effective tools to assess differences in doctors' behaviour and practice. ${ }^{10-12}$

A pool of 128 vignettes that made clinical sense were constructed: 96 for ovarian, eight for endometrial, and 24 for cervical cancer (these proportions were used because each cancer had a different number of patient factors [clinical variables] and the vignettes reflected this number). The vignettes depicted a combination of clinical details including: physical presentations such as lumps, masses, lesions, ulcers, irregular bleeding or postcoital bleeding; results of investigations such as the cancer antigen 125 (CA125) blood test for ovarian cancer, biopsies and ultrasound; duration of symptoms and the age of the patient. Each GP received a random allocation of 12 vignettes. At the end of each vignette, GPs were asked whether they would refer the patient, and if so, to whom. They were also asked to estimate the probability that the patient in each scenario had cancer.

The survey also collected the following data on the GPs: (i) demographic data such as age, sex, country of basic medical training, teaching experience and years of experience; (ii) practice location (urban versus rural) and practice type (solo versus group); (iii) information relating to access to gynaecologists, gynaecological oncologists and cancer multidisciplinary teams (MDTs); and (iv) the number of patients seen in the previous 5 years with potential gynaecological cancer.
We used a randomised incomplete block design (RIBD) for the study, which allows a limited number of vignettes to be presented to each participant, $^{13,14}$ thus lightening the burden on respondents. The main disadvantage of the RIBD is that data analysis is more complex and regression results need to be interpreted with caution. ${ }^{14}$ The RIBD also results in some loss of precision when estimating associations between patient factors and referral. ${ }^{14}$ Some interactions may also be confounded with between-subjects factors. ${ }^{7}$ This limitation was accounted for during analysis.

\section{Data collection}

From a population of over 20000 GPs (listed as currently practising, but limited to only one GP per practice), the Australasian Medical Publishing Company provided a random list of 5000 GPs, stratified by location with 2500 each from metropolitan and rural or remote areas. We sent participation packages to the first 3180 of these 5000 GPs (1615 metropolitan and 1565 rural GPs).

Data were collected between 1 April and 31 August 2009 using a mixed method, whereby participants could respond either by post (paper questionnaire) or on the internet (online questionnaire). The online and paper questionnaires were pilottested with members of the project reference group (invited clinicians, academics and consumer representatives) and staff of Cancer Australia. Modifications were made to both versions before the start of data collection. An upfront \$20 gift card was offered to a subset of GPs as an incentive to participate, and techniques such as getting a well respected peer to sign the cover letter, highlighting the uniqueness of each survey and sending up to three reminder letters to encourage participation were used. ${ }^{15,16}$

\section{Statistical analysis}

Data were loaded into a statistical software package (SPSS, version 13, SPSS Inc, Chicago, Ill, USA). $\chi^{2}$ tests were used to identify significant differences in referral practices between metropolitan and rural GPs (significant at $P<0.05)$. Logistic regression was used to determine the factors that were the strongest predictors of referral. To improve the precision of the regression models, the sample was adjusted for clustering (should referral patterns of individual clinicians be more similar than those across clinicians); stratification (should referral patterns within a stratum be more similar than across strata) and the finite population correction.

\section{Ethics and other approvals}

The study was approved by the University of Adelaide Human Research Ethics Committee, and the Australian Bureau of Statistics Statistical Clearing House.

\section{Results}

Of the 3180 GPs recruited to the study, 98 were ineligible or not contactable. Of the remaining 3082, 1402 responded (689 metropolitan and 713 rural or remote,) giving an overall response rate of $45.5 \%$. Eighty-two GPs whose location was identified in our original sample as rural classified themselves as practising in a metropolitan location; these were reclassified as metropolitan for the analysis, giving final numbers of 771 metropolitan and 631 rural GPs.

Compared with labour force statistics in $2008,{ }^{17}$ the study sample was generally representative in terms of sex and age distribution, with a small underrepresentation of GPs aged under 35 years and from $45-54$ years, and a slight overrepresentation of GPs aged over 55 years. Stratification by location ensured appropriate numbers for the intended comparisons between metropolitan and rural GPs; $56.0 \%$ practised in metropolitan regions and the remaining $45.0 \%$ in rural locations.

\section{Overall}

For vignettes identified as describing a woman with a high probability of cancer, $75.0 \%$ were referred by metropolitan GPs compared with $72.9 \%$ by their rural counterparts. GPs' referral decisions were closely aligned to their own estimation of cancer risk based on the patient factors present in each scenario. However, the mean estimations of cancer risk made by participating GPs did not always mirror the referral deci- 


\section{Predictors of a patient being referred for endometrial cancer by metropolitan and rural general} practitioners

Predictor

Age of patient 63 years

(reference group, 30 years)

Metropolitan
Rural

Thickened endometrium on pelvic ultrasound

(reference group, normal endometrium)

$\begin{array}{llll}\text { Metropolitan } & 2.052 & <0.001 & 7.8(4.5-13.6) \\ \text { Rural } & 1.473 & <0.001 & 4.4(2.5-7.5) \\ \begin{array}{l}\text { Duration of symptoms 8 weeks } \\ \text { (reference group, 2 weeks) }\end{array} & & & \\ \quad \text { Metropolitan } & 0.549 & 0.046 & 1.7(1.0-3.0) \\ \text { Rural } & 0.515 & 0.046 & 1.7(1.0-2.8)\end{array}$

* Unstandardised regression coefficient. † Relates to the predictor (age, condition of endometrium, duration of symptoms). Model evaluation for metropolitan GPs - Likelihood ratio test, $\chi^{2}(\mathrm{f}=3)=215.461(P<0.001)$; Hosmer-Lemeshow goodness of fit test, $\chi^{2}(\mathrm{df}=0.946(P=0.946)$; overall percentage correctly classified: $83.3 \%$.

Model evaluation for rural GPs - Likelihood ratio test, $\chi^{2}$ (df=3) $=248.176(P<0.001)$; Hosmer-Lemeshow goodness of fit test, $\chi_{(\mathrm{df}=6)}=2.664(P=0.850)$; overall percentage correctly classified: $82.5 \%$. sion provided in the unpublished Monash University report (based on the clinical practice guidelines and expert opinion available at the time of the pilot study ${ }^{6}$ ). Overall, GPs were significantly more likely to refer to a gynaecologist than to a gynaecological oncologist.

\section{Endometrial cancer}

For vignettes describing women identified as having a high probability of endometrial cancer, $67.7 \%$ resulted in a referral, of which $95.4 \%$ were to a gynaecologist. Metropolitan GPs were significantly more likely to indicate a decision to refer than rural GPs $(68.4 \%$ versus $61.1 \%)$. The best pre- dictors of referral for endometrial cancer were the age of the patient, results of pelvic ultrasound and duration of symptoms (Box 1).

\section{Ovarian cancer}

For vignettes describing women identified as having a high probability of ovarian cancer, $83.3 \%$ resulted in a referral, of which $70.8 \%$ were to a gynaecologist. The best predictors of referral for ovarian cancer were findings on physical examination, CA125 test results, ultrasound findings, duration of symptoms, age of the patient and years of experience of the GP (Box 2). Practitioners with more than 15 years of experience were more

\section{Predictors of a patient being referred for ovarian cancer by a general practitioner}

\begin{tabular}{lccc} 
Predictor & B* & $P$ & Adjusted odds ratio (95\% Cl) \\
\hline $\begin{array}{l}\text { Palpable mass on physical examination } \\
\text { (reference group, no abnormalities) }\end{array}$ & 3.484 & $<0.001$ & $32.6(25.5-41.8)$ \\
$\begin{array}{l}\text { Markedly elevated CA125 level } \\
\text { (reference group, normal level) }\end{array}$ & 2.888 & $<0.001$ & $18.0(14.5-22.2)$ \\
$\begin{array}{l}\text { Complex cyst on ultrasound } \\
\text { (reference group, no abnormalities) }\end{array}$ & 1.892 & $<0.001$ & $6.6(5.6-7.9)$ \\
$\begin{array}{l}\text { Duration of symptoms 8 weeks } \\
\text { (reference group, 2 weeks) }\end{array}$ & 0.767 & $<0.001$ & $2.2(1.9-2.5)$ \\
$\begin{array}{l}\text { Age of patient 58 years } \\
\text { (reference group, 35 years) }\end{array}$ & 0.495 & $<0.001$ & $1.6(1.4-1.9)$ \\
$\begin{array}{l}\text { Year of experience } \\
\text { (reference group, 1-15 years) }\end{array}$ & & & $1.1(0.9-1.4 \dagger)$ \\
$\quad 16-30$ years & 0.388 & 0.001 & $1.6(1.3-2.1)$ \\
\hline$\geqslant 31$ years & 0.484 & $<0.001$ & \\
\hline
\end{tabular}

CA125 $=$ cancer antigen $125 *$ Unstandardised regression coefficient. + Not significant

Model evaluation - Likelihood ratio test, $\chi^{2}$ (df=7) $=3730.431(P<0.001)$; Hosmer-Lemeshow goodness of fit test,

$\chi_{(\mathrm{df}=8)}^{2}=61.989(P<0.001)$; overall percentage correctly classified: $89.7 \%$. likely to refer a patient than those with less experience.

\section{Cervical cancer}

Almost $80 \%$ of vignettes describing women with a high probability of cervical cancer resulted in a decision to refer. Of these decisions, $90 \%$ were to refer to a gynaecologist. The best six predictors of referral for cervical cancer were the presence of a lesion, the age of the patient, irregular bleeding, duration of symptoms and postcoital bleeding (Box 3).

\section{GPs' access to services}

Overall, metropolitan GPs had significantly greater access to both private and public gynaecological oncologist services than rural GPs. This difference also extends to their access (and, by default, their patients' access) to gynaecological oncologist services associated with a multidisciplinary team (MDT). That is:

- $80 \%$ of metropolitan GPs and $50 \%$ of rural GPs reported access to private gynaecological oncologist services;

- $40 \%$ of metropolitan GPs and $25 \%$ of rural GPs reported that these private services were associated with an MDT;

- $80 \%$ of metropolitan GPs and $58 \%$ of rural GPs reported access to public gynaecological oncologist services; and

- $63 \%$ of metropolitan GPs and $40 \%$ of rural GPs reported that these public services were associated with an MDT.

\section{Discussion}

A significantly higher proportion of metropolitan GPs (compared with rural GPs) reported intentions to refer patients who had presentations indicative of endometrial cancer. In all other respects, referral patterns for urban and rural GPs were similar, although rural GPs had less access to gynaecological oncologists and MDTs than urban GPs. Metropolitan GPs were also significantly more likely than rural GPs to report private and public specialist services as being associated with an MDT. Overall, public services were more likely than private services to be associated with an MDT. Given that access to an MDT 
is recognised as best practice in the treatment of cancer, ${ }^{16,17}$ it is of concern that about $60 \%$ of metropolitan GPs reported not having access to a private gynaecological oncologist service associated with an MDT, and $37 \%$ in the public sector did not have such access. Higher proportions of rural GPs did not have access to private $(75 \%)$ and public (60\%) gynaecological oncologist services associated with an MDT. Consistent with the findings of other studies, ${ }^{18}$ we found that rural GPs and their patients have fewer options for oncology services than their urban counterparts. Better access to specialist services for referral could, in part, explain why metropolitan GPs were significantly more likely to refer patients with particular gynaecological conditions.

Our study shows that patient factors such as symptoms and clinical findings are better predictors of referral behaviour than the demographic characteristics of GPs. Across the three cancers, the age of the patient and the duration of symptoms were important determinants of referral, with specific factors such as ultrasound results, CA125 test results, bleeding, and ulcers or lesions being factors for individual cancers. Overall, the only clinician factor that was predictive of referral was years in practice, with more years in practice being associated with a higher likelihood of referral for ovarian cancer. Reasons for this difference are not clear. It appears that GPs' referral decisions are also closely aligned to their estimation of cancer risk based on the patient factors present in each vignette. This variance in the assessment of cancer risk could, in part, explain the variance in referral practice.

For endometrial cancer, for which there are no current Australian guidelines, there was greater variation in referral practices: $68 \%$ of vignettes deemed to have a high probability of cancer were referred compared with $83 \%$ for ovarian cancer and $80 \%$ for cervical cancer, for which Australian guidelines are available.

This study had a number of limitations. First, measuring referral based on hypothetical scenarios, regardless of the validity of the included variables, can only provide evidence of practitioner intention, not actual prac-

\begin{tabular}{|c|c|c|c|}
\hline Predictor & B* & $P$ & Adjusted odds ratio $(95 \% \mathrm{Cl})$ \\
\hline $\begin{array}{l}\text { Lesion on cervix } \\
\text { (reference group, no lesion) }\end{array}$ & 1.791 & $<0.001$ & $6.0(4.7-7.6)$ \\
\hline $\begin{array}{l}\text { Age of patient } 71 \text { years } \\
\text { (reference group, } 32 \text { years) }\end{array}$ & 1.783 & $<0.001$ & $6.0(4.8-7.4)$ \\
\hline $\begin{array}{l}\text { Irregular bleeding } \\
\text { (reference group, no irregular bleeding) }\end{array}$ & 0.667 & $<0.001$ & $1.9(1.5-2.5)$ \\
\hline $\begin{array}{l}\text { Duration of symptoms } 8 \text { weeks } \\
\text { (reference group, } 2 \text { weeks) }\end{array}$ & 0.654 & $<0.001$ & $1.9(1.6-2.3)$ \\
\hline $\begin{array}{l}\text { Postcoital bleeding } \\
\text { (reference group, no bleeding) }\end{array}$ & 0.460 & 0.008 & $1.6(1.2-2.1)$ \\
\hline
\end{tabular}

* Unstandardised regression coefficient.

Model evaluation - Likelihood ratio test, $\chi^{2}(\mathrm{df}=5)=637.460(P<0.001)$; Hosmer-Lemeshow goodness of fit test,

$\chi^{2}(\mathrm{df}=8)=46.278(P<0.001)$; overall percentage correctly classified: $80.4 \%$.

tice. Second, the respondents' capacity to make referral decisions was restricted by the factors in the vignettes, so the reported referral rates could underestimate actual referral rates had clinicians been able to consider other forms of investigation before making a referral decision. Third, vignette scenarios rarely include minor symptoms that might provide additional information to help with decision making. ${ }^{8,9}$ However, when using a comparison with standardised patients (the gold standard for measuring quality of clinical practice), vignettes have been found to be a more accurate method than medical record abstraction. ${ }^{8}$ Finally, a response rate of less than $60 \%$ may limit the generalisability of our results.

Overall, our findings highlight the variability in Australian GPs' referral practices in relation to women with a range of gynaecological symptoms attributable to endometrial, ovarian or cervical cancer. Greater awareness, understanding, and adherence to available national and international guidelines could help standardise referral behaviour. However, evidence-based clinical practice guidelines for endometrial cancer still need to be developed and, in light of our results, current guidelines for ovarian and cervical cancer should be reviewed. ${ }^{7}$ Unfortunately, the existence of evidence-based clinical practice guidelines does not guarantee that they will be used in daily practice. Changing the behaviour of physicians is complex and difficult, and interventions developed to change their behaviour have shown limited effects. ${ }^{19}$ Identifying efficient implementation strategies to increase the uptake of guideline recommendations will be a major challenge for the future. Our findings also have implications for current training curricula and continuing professional development.

Acknowledgements: This research was funded by the National Centre for Gynaecological Cancers at Cancer Australia. We thank Julie Mueller, Susan Hanson and Professor David Currow for their advice, support and contribution to the study. We also thank all members of the Project Reference Group, especially Professor lan Hammond of the Royal Australian and New Zealand College of Obstetricians and Gynaecologists, for their invaluable assistance during the data collection phase. We also thank Jeremy Bennett of the Hunter Valley Research Foundation, who programmed and managed the online survey, and Kim Bridge and Zena McDonald for entering data from the mail surveys. Finally, we thank all the GPS who participated in the study.

Competing interests: No relevant disclosures.

Received 9 Aug 2010, accepted 29 Aug 2011.

1 Australian Institute of Health and Welfare and the Australasian Association of Cancer Registries. Cancer in Australia: an overview, 2008. Canberra: AlHW, 2008. (AlHW Cat. No. CAN 42; Cancer Series No. 46.) http://www.aihw.gov.au/ publication-detail/?id=6442468196 (accessed Oct 2011).

2 Marsden DE, Friedlander M, Hacker NF. Current management of epithelial ovarian carcinoma: a review. Semin Surg Oncol 2000; 19: 11-19.

3 Jayde V, White K, Blomfield P. Symptoms and diagnostic delay in ovarian cancer: a summary of the literature. Contemp Nurse 2009-10; 34: 55-65.

4 Barry J, Breen N. The importance of place of residence in predicting late-stage diagnosis of breast or cervical cancer. Health Place 2005; 11 : 15-29.

5 Piterman L, Koritsas S. Part II. General practitioner-specialist referral process. Intern Med J 2005; 35: 491-496.

6 Britt H, Millar GC, Knox S, et al. BEACH (Bettering the Evaluation And Care of Health). General practice activity in Australia 2006-2007. Canberra: Australian Institute of Health and Welfare, 2008. (AlHW Cat. No. GEP 21.) http:// www.aihw.gov.au/publication-detail/?id= 6442468067 (accessed Oct 2011).

7 Jiwa M, Gordon M, Arnet H, et al. Referring patients to specialists: a structured vignette survey of Australian and British GPs. BMC Family Pract 2008; 9: 2.

8 National Breast Cancer Centre. Clinical Practice Guidelines for the management of women with epithelial ovarian cancer. Sydney: NBCC, 2004. http://www.nhmrc.gov.au/publications/ synopses/_files/cp98.pdf (accessed Oct 2011).

9 National Cervical Screening Program. Screening to prevent cervical cancer: guidelines for the management of asymptomatic women with screen detected 
abnormalities. Canberra: National Health and Medical Research Council, 2005. http:// www.nhmrc.gov.au/publications/synopses/_ files/wh39.pdf (accessed Oct 2011).

10 Peabody JW, Luck J, Glassman P, et al. Measuring the quality of physician practice by using clinical vignettes: a prospective validation study. Ann Intern Med 2004; 141: 771-780.

11 Dresselhaus TR, Peabody JW, Luck J, Bertenthal D. An evaluation of vignettes for predicting variation in the quality of preventive care. J Gen Intern Med 2004; 19: 1013-1018.

12 Veloski J, Tai S, Evans AS, Nash DB. Clinical vignette-based surveys: a tool for assessing physician practice variation. Am J Med Qual 2005; 20: 151-157.

13 West P. Reproducing naturally occurring stories: vignettes in survey research. Glasgow: MRC Social and Public Health Sciences Unit, 1982.

14 Graham ME, Cable DM. Consideration of the incomplete block design for policy-capturing research. Organizat Res Meth 2001; 4: 26-45.

15 VanGeest JB, Johnson TP, Welch VL. Methodologies for improving response rates in surveys of physicians: a systematic review. Eval Health Prof 2007; 30: 303-321.

16 Edwards P, Roberts I, Clarke M, et al. Methods to increase response rates to postal questionnaires. Cochrane Database Syst Rev 2009; (3): MR000008.

17 Australian Institute of Health and Welfare. Medical labour force 2008. Bulletin 82, 13 October 2010. Canberra: AlHW, 2010. (AlHW Cat. No. AUS 131.) http://www.aihw.gov.au/ publications/aus/bulletin82/12016.pdf (accessed Oct 2011).

18 Junor EJ, Hole DJ, Gillis CR. Management of ovarian cancer: referral to a multidisciplinary team matters. Br J Cancer 1994; 70: 363-370.

19 Knight JA. Change management in cancer care: a one-stop gynaecology clinic. Br J Nursing 2007; 16: 1122-1126. 